\title{
Giemsa staining for cysts and trophozoites of Pneumocystis carinii
}

\author{
J WALKER, G CONNER, J HO, C HUNT, L PICKERING Medical Parasitology Unit, \\ Department of Infectious Diseases and Microbiology, Westmead Hospital, Australia
}

SUMMARY Although Giemsa staining has been routinely used for the detection of trophozoites and intracystic bodies in smears of bronchoalveolar lavage fluid (BAL) from patients with Pneumocystis carinii pneumonia, it does not normally stain the cyst wall. For detection of the cysts other stains such as toluidine Blue ' $O$ ' and methenamine silver must be used as well. Sulphation of smears before staining with Giemsa allows cysts to be visualised, thus enabling a single stain to be used to show all the stages of BAL or sputum, which is particularly useful, considering the increase in the prevalence of $P$ carinii pneumonia in conjunction with the spread of AIDS.

Pneumocystis carinii pneumonitis has increased in prevalence in parallel with the epidemic of acquired immune deficiency syndrome (AIDS). As a consequence laboratories are receiving increasing numbers of requests for the diagnosis of $\boldsymbol{P}$ carinii, normally in induced sputum or bronchoalveolar lavage fluid (BAL). Of the various stains used for the recognition of $P$ carinii in these fluids or in lung impression smears, those which stain the cyst wall are most commonly applied. They include modifications of the GrocottGomori methanamine silver stain, toluidine blue $\mathrm{O}$ and Gram's stain. ${ }^{1}$ The trophozoites and intracystic bodies in intact cysts can be stained with Giemsa, but the cyst wall does not take up this stain.

Interpretation of the results of Giemsa staining requires experience and skill if satisfactory degrees of sensitivity and specificity are to be obtained, particularly if there are large numbers of bacteria in the specimen. The results of any of these methods of staining can be improved if the smears used are prepared by cytocentrifugation ${ }^{2}$ and if dithiothreitol (Sputolysin; Behring Diagnostics) is used to reduce the density of the material on the slide. ${ }^{3}$ Hughes suggested that at least three of these stains should be used for all specimens and while this may not be a problem for most hospital laboratories, it may not be possible in smaller units. A single stain which would make both cysts and trophozoites visible would be a valuable addition to the technique for diagnosis of Pneumocystis.

\section{Material and methods}

Induced sputum specimens from patients suspected of Accepted for publication 3 November 1988 having $P$ carinii pneumonitis were mixed with an equal volume of dithiothreitol (Sputolysin; Behring Diagnostics), vortexed briefly, and then incubated at $37^{\circ} \mathrm{C}$ for 15 minutes. After incubation the specimen is mixed with an equal volume of phosphate buffered saline, vortexed again, then centrifuged at $1300 \times g$ for five minutes. The resulting pellet is spread on to microscope slides, heat fixed, sulphated and stained with Giemsa or Field's stain. Bronchoalveolar lavage fluid specimens were concentrated by cytocentrifugation (Shandon Cytospin II) without Sputolysin treatment. The reagents used were:

1 Sulphation reagent $15 \mathrm{ml}$ of concentrated sulphuric acid is added slowly, by glass pipette, to $45 \mathrm{ml}$ of glacial acetic acid in a Coplin jar. The Coplin jar should be standing in a container of cool tap water (not below $10^{\circ} \mathrm{C}$ ). The solution is gently mixed with a glass rod and the jar sealed with petroleum jelly. The reagent can be used for one week if kept at room temperature.

2 Giemsa stain 10\% Giemsa (Gurr's improved R66) in phosphate buffer ( $\mathrm{pH} \mathrm{7 \cdot 2).}$

\section{STAINING PROCEDURE}

Ethanol or heat fixed BAL smears were placed in the sulphation reagent (using forceps) for 10 minutes, then removed, and washed in running tap water for five minutes. The slides are then placed, smear side down, in a small dish of the Giemsa stain and left for 30 minutes. After further washing to remove excess stain the slides are then allowed to air dry. When thoroughly dry a coverslip can be permanently mounted using any suitable mountant. If a low power oil immersion objective is available the slide can be scanned without adding a coverslip. 


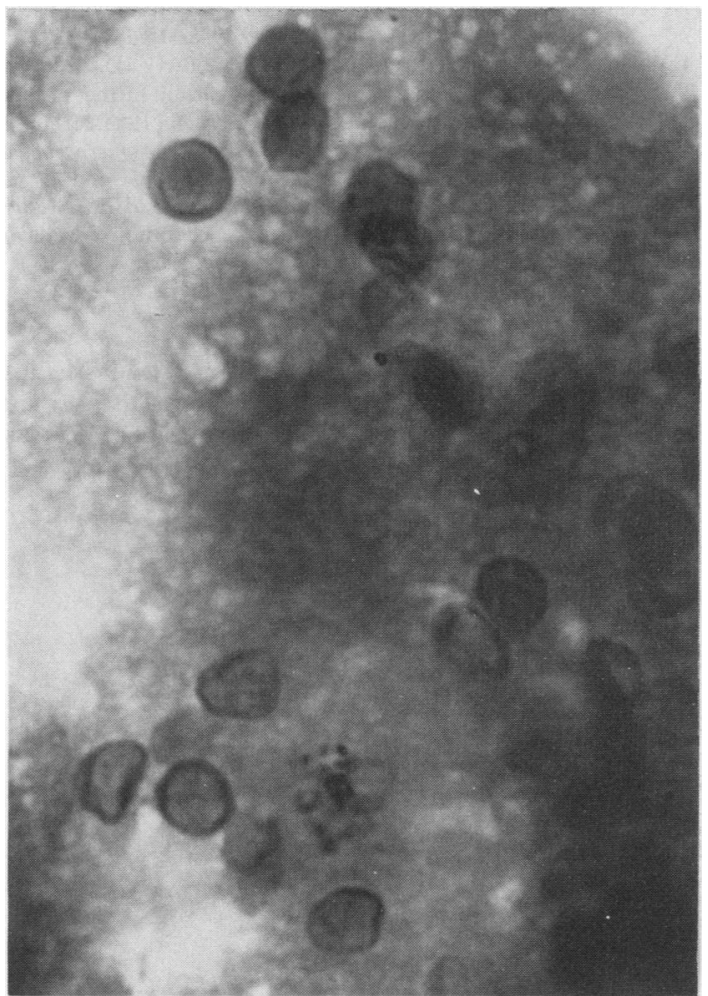

Fig 1 Cysts of Pneumocystis carinii stained with Giemsa after sulphation.

\section{Results}

The appearance of $\boldsymbol{P}$ carinii cysts stained by this technique is similar to that obtained following the use of toluidine blue $\mathrm{O}$ stain $^{4}$ (fig 1). The cysts are oval to circular, about $5 \mu \mathrm{m}$ in diameter. The outline is generally magenta and the central portion of the cyst lavender, though the exact colour varies from place to place in the smear with the red tints predominating in some areas and the blue in others. A potential source of error with this stain, as with toluidine blue, is the frequent presence of yeasts, including non-budding forms, in sputum samples and, occasionally, in BAL. In bronchoalveolar lavage fluid smears stained with Giemsa without prior sulphation, the free trophozoites, which have blue cytoplasm and purple nuclei, are seen embedded in the frothy honeycombed material which is also prominent in preparations stained with Papanicolaou stain. This "frothy exudate" stains magenta with Giemsa. In these smears the intracystic bodies ("sporozoites") stain violet while cyst walls are only faintly visible.

Field's stain (Gurr), normally used for staining thick films for diagnosis of malaria, ${ }^{5}$ can be applied in

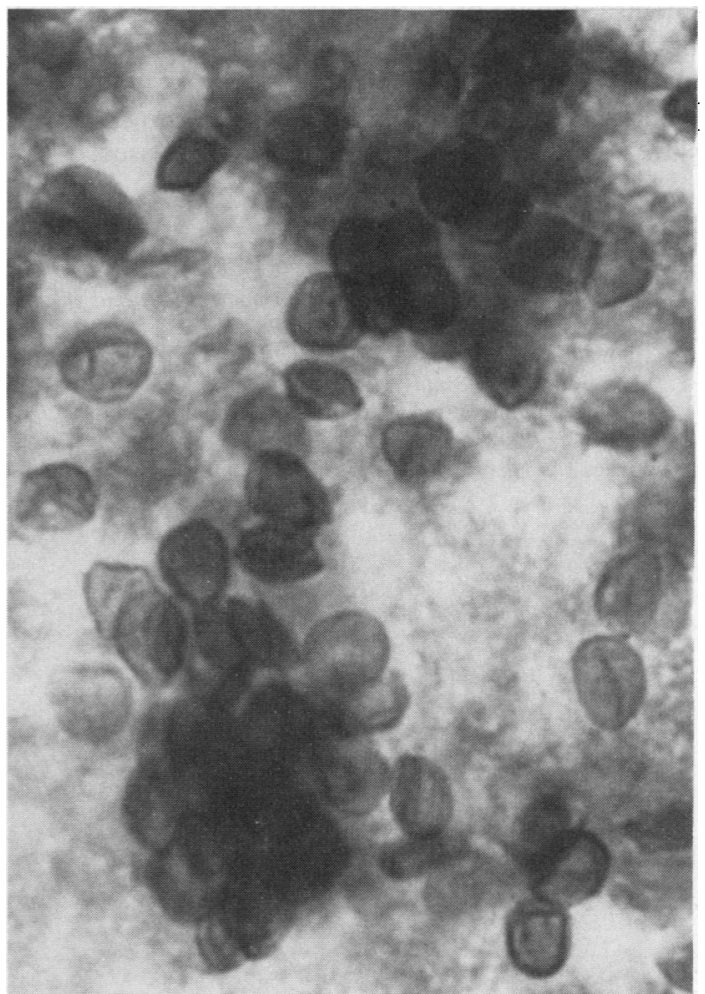

Fig 2 Cysts of Pneumocystis carinii stained with Field's stain after sulphation.

the same way as Giemsa for staining Pneumocystis. This two part stain has the advantage of rapidity; the total staining time is about 10 to 15 seconds. The smears, whether sulphated or not, are placed in Field's A solution (active components methylene blue and azur I) for about five seconds, then washed in water before being dipped into Field's B (active component eosin [yellow, water soluble]) for one second. After the excess stain is quickly rinsed off the smears are air dried before coverslipping. The appearance of $P$ carinii is similar to that after Giemsa staining (fig 2).

\section{Discussion}

Sulphation apparently modifies the surface of $P$ carinii cysts in a way which enables polychrome stains such as Giemsa to react. The change is not simply due to the exposure of cysts by removal of the "honeycomb"-like alveolar exudate, because this is still present, though its staining reaction is modified. The large numbers of trophozoites normally embedded in this exudate seem to be destroyed by sulphation.

The ability to show both cysts and trophozoites of $P$ carinii with a single, widely available stain such as 
Giemsa, should be to the advantage of small diagnostic laboratories and the rapidity of the Field's stain method of value to all. With all the staining methods mentioned it is desirable to include a known positive slide with every batch of specimens processed.

\section{References}

1 Hughes WT. 1987. Diagnosis. In: Pneumocystis carinii pneumonitis. Vol II. Florida: CRC Press, 1987:35-71.

2 Gill VJ, Evans G, Stock F, Parrillo JE, Masur H, Kovacs JA. Detection of Pneumocystis carinii by fluorescent-antibody stain using a combination of three monoclonal antibodies. J Clin Microbiol 1987;25:1837-40.

3 Kovacs JA, Ng VL, Masur H, et al. Diagnosis of Pneumocystis carinii pneumonia: improved detection in sputum with use of monoclonal antibodies. $N$ Engl J Med 1988;318:589-93.

4 Witebsky FB, Andrews JWB, Gill VJ, MacLowry JD. Modified toluidine blue $\mathrm{O}$ stain for Pneumocystis carinii: further evaluation of some technical factors. J Clin Microbiol 1988;26:774-5.

5 Field JW, Sandosham AA, Yap Loy Fong. The microscopical diagnosis of human malaria I. A morphological study of the erythrocytic parasite in thick blood films. Second Ed. Studies from the Institute for Medical Research, Federation of Malaya, No 30. Kuala Lumpur: Economy Printers, 1963.

Requests for reprints to: Dr J Walker, Head Medical Parasitology Unit, Department of Infectious Diseases and Microbiology, Westmead Hospital, Westmead 2145, Australia. 